\title{
Synthesis and characterization of Ag@polycarbazole nanoparticles using different oxidants and their dispersion behavior
}

\author{
ZAHOOR AhMAD $^{1 *}$, M.A Choudhary ${ }^{1}$, M.A. Mirza ${ }^{1}$, J.A. Mirza ${ }^{2}$ \\ ${ }^{1}$ Department of Chemistry, Mirpur University of Science and Technology (MUST), Mirpur, AJ\&K, Pakistan \\ ${ }^{2}$ Department of Material and Metallergy, Pakistan Institute of Engineering and Applied Sciences (PIEAS), \\ Nilore, Islamabad, Pakistan
}

\begin{abstract}
Ag nanoparticles (NPs) encapsulated by polycarbazole (PCz) have been synthesized using ion adsorption method. The PCz synthesis around $\mathrm{Ag}$ NPs has been performed by adsorbing $\mathrm{Ag}^{+1}$ and $\mathrm{Fe}^{+3}$ oxidants onto $\mathrm{Ag} \mathrm{NPs}$, which initiated surface polymerization and thus, Ag NPs@PCz nanocomposite has been synthesized. The morphology of pure NPs and composite NPs was characterized by TEM which also elucidated the effect of oxidant on the core NPs, beside their morphologies and phase contrasts of metal NPs and polymer. The polymer around the surface of core NPs was characterized by FT-IR which proved that PCz was the organic phase of the composite NPs. UV-Vis spectroscopy has been employed to study surface plasmon resonance (SPR) of pure NPs and composite of NP which demonstrated that SPR of core NPs remained preserved after coating with the polymer. Furthermore, Zeta Sizer Nano series has been applied to analyze the dispersion behavior of pure NPs and composite NPs which displayed the greatly improved dispersion behavior of the composite NPs as compared to pure Ag NPs. Therefore, our study proved helpful to analyze the suitability of metal oxidants for PCz based nanocomposite synthesis and determination of their optical and dispersion behavior.
\end{abstract}

Keywords: Ag@PCz; nanocomposites; optical behavior; dispersion trend

(C) Wroclaw University of Technology.

\section{Introduction}

Polymer-nanometal matrices and nanometalpolymer composites are potential materials for many technological applications due to their unique physical, and photo-electrochemical properties [1]. Nanocomposite materials have considerable potential for the fabrication of sensors in the context of environmental and food safety monitoring [2]. The metal-polymer nanocomposites have also applications which use their ability to detect metal ions in aqueous solutions due to enhanced surface area of polymer [3]. Moreover, conjugated polymers possess electrical and luminescence peculiarities and magnitude of such type of properties can be enhanced using nanometallic components within the polymer matrix [4]. Among the conductive composite materials, silver is the most often used because of its high electrical conductivity and

\footnotetext{
*E-mail: zahoorbuct@hotmail.com
}

oxidation resistance. In this way, nano $\mathrm{Ag}$ is a potential material to increase electrical and luminescent properties of encasing polymers.

Among the conductive and luminescent polymers, $\mathrm{PCz}$ bears special significance due to the electron and hole transporting behavior that enables its utilization in organic light emitting diodes and electrochromic displays [5]. It is also a photorefractive material possessing nonlinear optical properties which are promising to be used in modulators, electro-optic switches, and frequency doublers [6]. It is further very desirable to develop a sensor for metal ions, $\mathrm{CO}_{2}$ and $\mathrm{N}$, containing bases such as ammonia, amine, and nucleotides, having detection ability up to their trace level. Moreover, the polymer shows high thermal and environmental stability along with fiber and beads making ability [7]. Therefore, it was desirable to synthesize PCz based Ag nanocomposite to develop synergistic properties. 
Herein we used a strategy to adsorb $\mathrm{Ag}^{+1}$ and $\mathrm{Fe}^{+3}$ oxidants onto Ag NPs which were prepared by polyol reduction method to polymerize carbazole adhering to the surface of NPs [8]. The exploitation of $\mathrm{Ag}^{+1}$ and $\mathrm{Fe}^{+3}$ to polymerize carbazole around Ag NPs has been carried out to fabricate the composite materials. The morphology of the nanocomposite and polymer-metal phases was analyzed by TEM. The polymer was further characterized by FT-IR as PCz. Finally, surface plasmon resonance (SPR) of metal NPs and their dispersion behavior was explored. The data indicated the successful synthesis of $\mathrm{Ag}^{+1}$ and $\mathrm{Fe}^{+3}$ assisted $\mathrm{Ag} @ \mathrm{PCz}$ nanocomposites, their innate preservation, and finally their improved dispersion which aimed at maximum utilization of the nanostructures. Otherwise, agglomeration of pure NPs could be a cause to destroy the properties of nanodomain. The study also revealed the effect of corresponding anions over the metal nanostructure. The study would be helpful to make better selection of oxidizing salts, while considering the effect of anionic part over metal NPs. The report would promote the exploitation of $\mathrm{Ag} @ \mathrm{PCz}$ nanocomposites in the field of electro/photo conductivity, electric and optical chromic displays, and sensors for detection of metal ions, $\mathrm{CO}_{2}$ and $\mathrm{N}$ containing bases, such as ammonia, amine, and nucleotides.

\section{Materials}

$\mathrm{AgNO}_{3}$ (99.8\%), ethylene glycol (96\%), ethanol $(99.8 \%)$, acetone $(99.5 \%)$, and acetonitrile (95\%), were purchased from Beijing Shiji. PVP (K30) was bought from Fuchen Chemical Reagent Company, Tianjin. Carbazole monomer (95\%) was bought from Shanghai Zhixin Chemical Industry Co., Ltd., and was recrystallized in 1:1 of n-hexane/isopropanol solution before the use. Cupric acetate (98\%) was bought from Beijing Yili Chemical Reagent Company.

\section{Method}

The Ag@PCz nanocomposite was prepared in two steps, the first step was a synthesis of $\mathrm{Ag}$ NPs and the second step was a formation of $\mathrm{PCz}$ thin sheathing around the NPs to obtain Ag@PCz nanocomposites. The description of each step is given in the following sections.

\subsection{The synthesis of Ag nanoparticles}

The Ag NPs were prepared by polyol reduction method, where ethylene glycol (EG) was used as a solvent for making solutions and also as a reducing agent. According to the typical approach, $10 \mathrm{~mL}$ EG was refluxed for two hours at $130{ }^{\circ} \mathrm{C}$ to remove water content. Then $6 \mathrm{~mL}, 0.1 \mathrm{M}$ solution of $\mathrm{AgNO}_{3}$ and $6 \mathrm{~mL} 1.5 \mathrm{M}$ solution of PVP were added simultaneously into refluxed EG within 10 minutes. On addition, yellow color appeared which gradually turned into bluish gray within 15 to 20 minutes. The whole reaction system was kept at $130{ }^{\circ} \mathrm{C}$ for one hour. The product was washed and centrifuged several times using acetone and ethanol to remove PVP and EG. The washing process was repeatedly used, until the washing liquid appeared clear. In this way NPs were prepared and used for composite synthesis.

\subsection{Synthesis of $\mathrm{Ag} @ \mathbf{P C z}$ nanocomposite}

The oxidizing agents applied for Ag@PCz synthesis were $\mathrm{AgNO}_{3}$, and $\mathrm{FeCl}_{3}$, respectively. In case of $\mathrm{AgNO}_{3}$ as oxidizing agent, $0.1 \mathrm{~g} \mathrm{AgNO}_{3}$ was dissolved in $6 \mathrm{~mL}$ ethanol and mixed with Ag NPs prepared in step one by ultrasonication for 10 minutes. It was subsequently centrifuged to remove the non-adsorbed cations and anions. It has already been reported that $\mathrm{Ag}$ nanostructure can adsorb $\mathrm{Ag}^{+1}$ due to the common ions effect [9]. It was mixed with $5 \mathrm{~mL}, 0.1 \mathrm{M}$ carbazole solution in acetonitrile. The reaction was allowed to continue for 48 hours. Similarly, in case of $\mathrm{FeCl}_{3}$, which is very suitable oxidizing agent for carbazole polymerization, $0.1 \mathrm{~g} \mathrm{FeCl}_{3}$ was dissolved in $5 \mathrm{~mL}$ ethanol and mixed with Ag NPs. It was again centrifuged to remove non adsorbed compound and then mixed with $0.1 \mathrm{M}$ carbazole solution in acetonitrile. The reaction was allowed to continue for 48 hours. In both cases metal ions were adsorbed onto the Ag NPs and activated by surface initiated polymerization around NPs. In this way, both metal ions were found successful in preparing $\mathrm{Ag} @ \mathrm{PCz}$ 
nanocomposite. The material was washed with ethanol and deionized water, and dried for further analysis.

\section{Results and discussion}

The morphology of Ag NPs prepared at $130{ }^{\circ} \mathrm{C}$ was observed under TEM, which showed that the nanomaterial comprised of nanoparticles having diameter in the range of 60 to $70 \mathrm{~nm}$. These NPs were adsorbed by $\mathrm{Ag}^{+1}$ and $\mathrm{Fe}^{+3}$ ions and subsequently surface initiated polymerization of carbazole was accomplished. The pure Ag NPs and surface coated $\mathrm{Ag}$ NPs assisted by $\mathrm{Ag}^{+1}$ and $\mathrm{Fe}^{+3}$ ions are shown in Fig. 1. According to the data given in Fig. 1a, the Ag NPs produced by polyol reduction method are spherical with the diameters in the range of 60 to $70 \mathrm{~nm}$. Moreover, according to Fig. $1 \mathrm{~b}$ and $1 \mathrm{c}$, the nanocomposites were produced by $\mathrm{Ag}^{+1}$ and $\mathrm{Fe}^{+3}$ respectively. Both oxidizing agents proved successful in producing surface coating with the thicknesses of 5 to 8 and 10 to $12 \mathrm{~nm}$, respectively. Moreover, surface of Ag NPs remained intact when $\mathrm{Ag}^{+1}$ was used as oxidizing agent whereas its texture corroded when $\mathrm{Fe}^{+3}$ was used. It was due to some $\mathrm{Cl}^{-1}$ which has strong reactivity with Ag. It was also observed that during the polymerization, the NPs were also crosslinked in isolated form, i.e. without making agglomerations. Therefore, it was observed that $\mathrm{Ag}^{+1}$ and $\mathrm{Fe}^{3+}$ are effective oxidizing agents which could adsorb onto the surface of NPs and then polymerize the monomer around the surface of Ag nanostructures.

The polymerization of carbazole around $\mathrm{Ag}$ NPs by $\mathrm{Ag}^{+1}$ and $\mathrm{Fe}^{+3}$ was further characterized by FT-IR which is shown in Fig. 2. Both the spectra show N-H stretching at $3430 \mathrm{~cm}^{-1}$ and aromatic ring stretching at $1610 \mathrm{~cm}^{-1}$ of polymer [10]. The C-N stretching observed at $1450 \mathrm{~cm}^{-1}$ is very prominent in case of $\mathrm{Fe}^{+3}$ and weak in case of $\mathrm{Ag}^{+1}$. It might be due to the greater thickness of polymer surfactant when $\mathrm{Fe}^{+3}$ was used. The bands at 820 and $740 \mathrm{~cm}^{-1}$ are the bands of polymerization which reflect the polymerization from 3,6 position of carbazole [11]. In case of $\mathrm{Ag}^{+1}$ all bands are shifted toward the lower wavenumber. This may

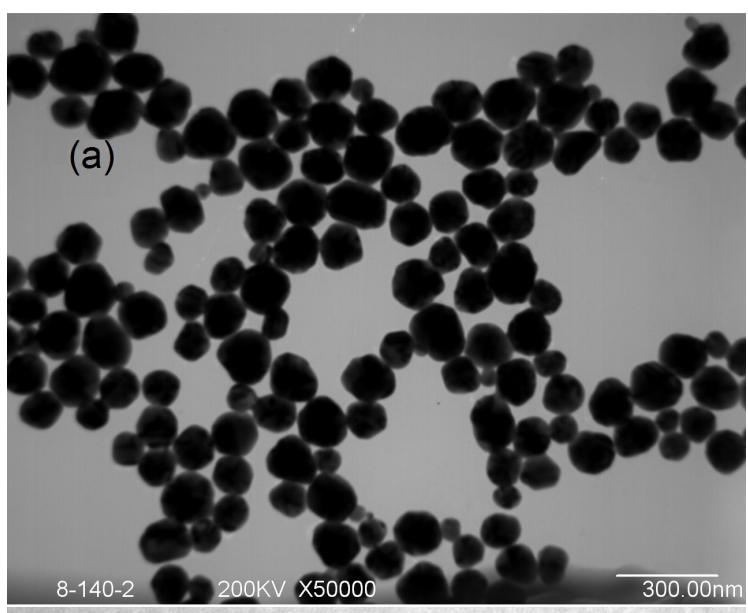

(b)
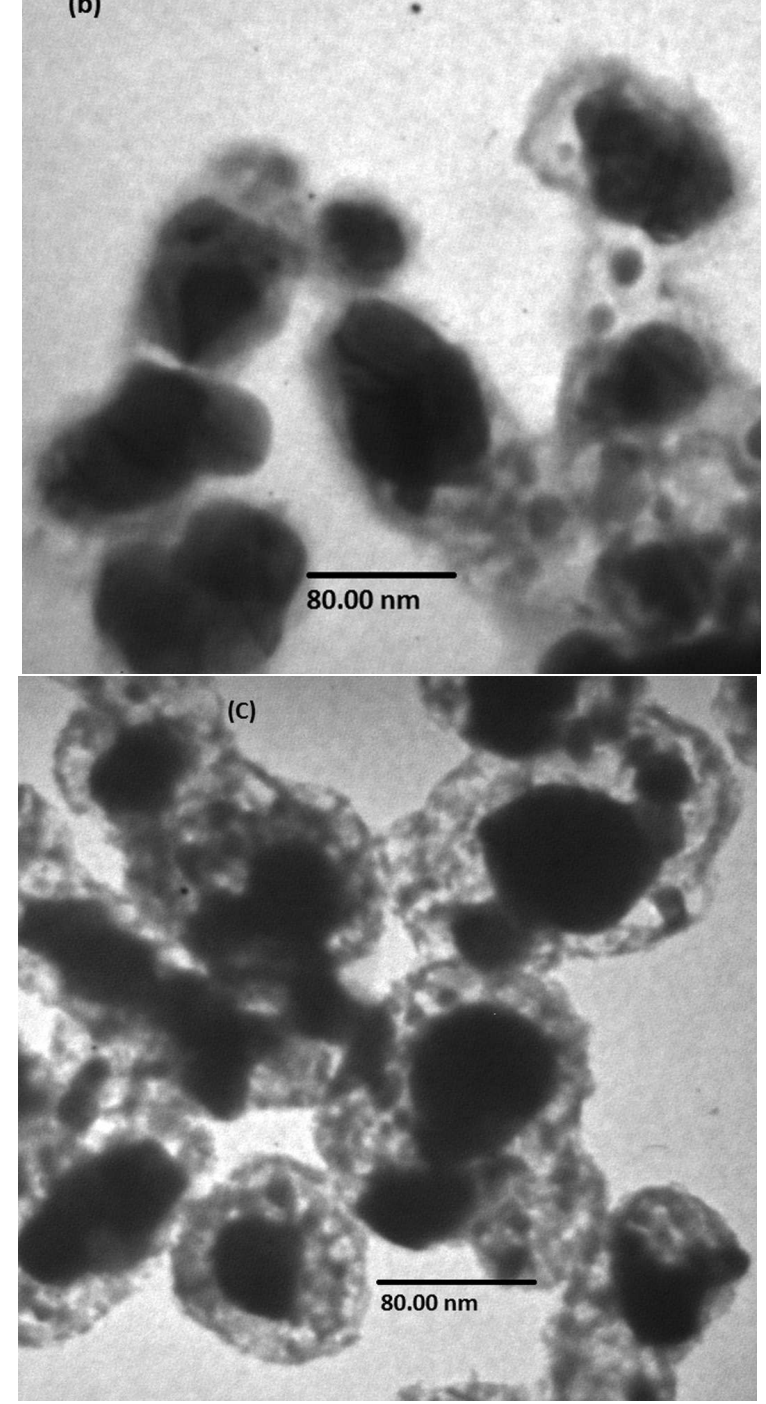

Fig. 1. TEM pictures of (a) pure Ag NPs (b) $\mathrm{Ag}^{+1}$, (c) $\mathrm{Fe}^{+3}$ based nanocomposite. 
be the effect of doping of metal ions. Thus, the FT-IR proves that $\mathrm{PCz}$ assisted by both metal cations has been fabricated over Ag nanostructures. Moreover, the composite would combine the properties of both components, which are thermal stability, resistance against oxidation, photo- and electroluminescence along with appropriate charge and plasma density of metal NPs.

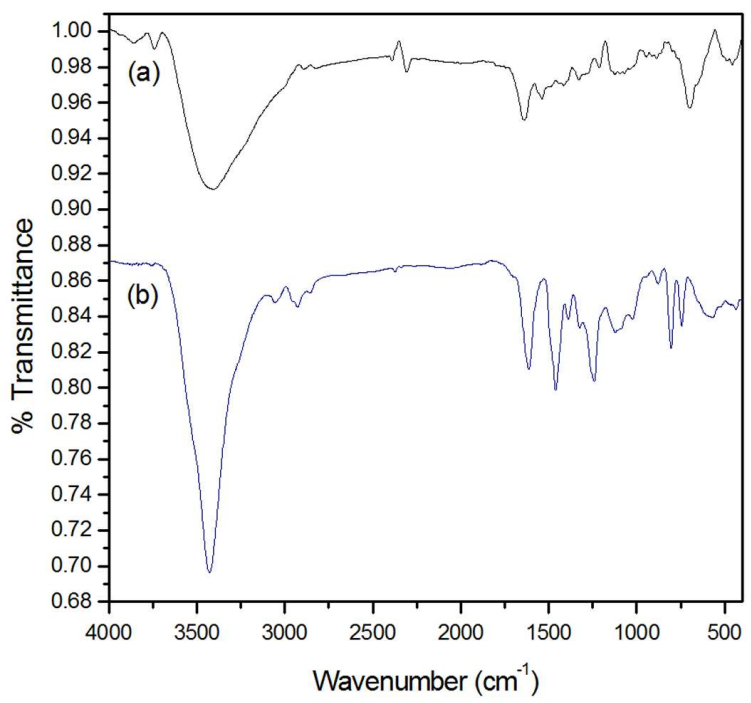

Fig. 2. FT-IR spectrum displaying PCz synthesized by (a) $\mathrm{Ag}^{+1}$ and (b) $\mathrm{Fe}^{+3}$ adsorbents.

The metal NPs display surface plasmon resonance (SPR), which is the basic characteristic of metal based NPs having diameter larger than $2.00 \mathrm{~nm}$ [12]. It is specifically related with dimension and nature. The SPR of Ag NPs and $\mathrm{Ag}^{+1}$ based nanocomposite are shown in Fig. $3 \mathrm{a}$ and the nanocomposite produced by $\mathrm{Fe}^{+3}$ is displayed in Fig. 3b. According to the data presented in Fig. 3, it can be realized that pure Ag NPs possess SPR behavior at $445 \mathrm{~nm}$ and it remains intact with reference to the position for carbazole polymerized by $\mathrm{Ag}^{+1}$, but slight decrease in intensity due to surface passivation is observed [8]. However, in case of $\mathrm{FeCl}_{3}$ the polymer thickness was found to be significantly increased which caused an intense decrease in SPR and blue shifting. In addition, TEM reflected the surface corrosion of $\mathrm{Ag}$ NPs which was due to the presence of $\mathrm{Cl}^{-1}$ ions in the reacting mixture that reacted with nano $\mathrm{Ag}$ and decreased its size, what resulted in the blue shift in SPR. However, the decrease in SPR intensity could also be related with the basic concept of surface passivation, which states that as the passivation is increased the SPR would decrease [13]. Moreover, in Fig. $3 b$ the $\pi-\pi^{*}$ transition of polymer at $293 \mathrm{~nm}$ is observed which is missing in Fig. 3a. It can be assumed that the $\pi-\pi^{*}$ transition was due to high density of polymer around Ag NPs [14]. From the SPR observation it was inferred that the polymer coating passivated the SPR of NPs with respect to its thickness. Moreover, the preservation of SPR in each case indicates that the metal nanostructures during polymerization maintained their nature and have not undergone to frequent oxidation. After surfactant coating their preservation against environmental oxidation was further improved as the SPR character remained intact. The strategy enabled us to utilize the material in composite form in light emitting diodes, solar cells and $\mathrm{PCz}$ based dyes [8]. Furthermore, by heating at 500 to $600{ }^{\circ} \mathrm{C}$ under inert atmosphere, the surfactant can fully be removed and pure NPs can be employed for their innate applications, such as antimicrobial, catalytic and enzyme immobilization [15].
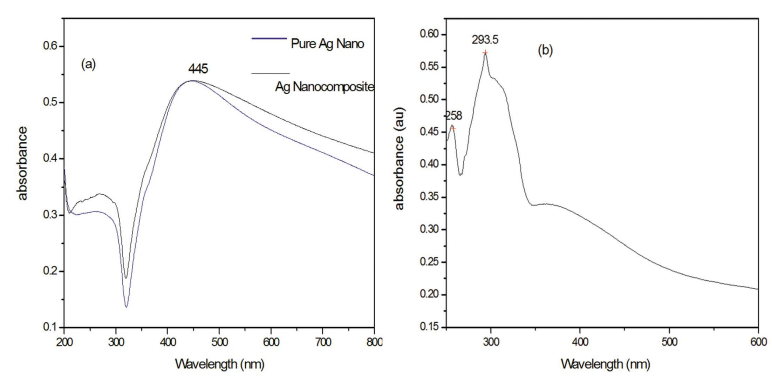

Fig. 3. SPR expression of (a) $\mathrm{Ag}$ NPs and $\mathrm{Ag}^{+}$ based PCz nanocomposite, (b) $\mathrm{Fe}^{+3}$ based $\mathrm{PCz}$ nanocomposites.

The polymers have varying degree of interaction with different solvents, and therefore can make dispersion, suspension or true solution. The interaction of $\mathrm{PCz}$ with common organic solvents such as methanol, ethanol, acetone and halogenated solvents is considerably greater as compared to many other polymers, like polypyrrole, polyannilin, polythiophene [16]. Therefore, the utilization of $\mathrm{PCz}$ as a surfactant is expected to considerably enhance the 

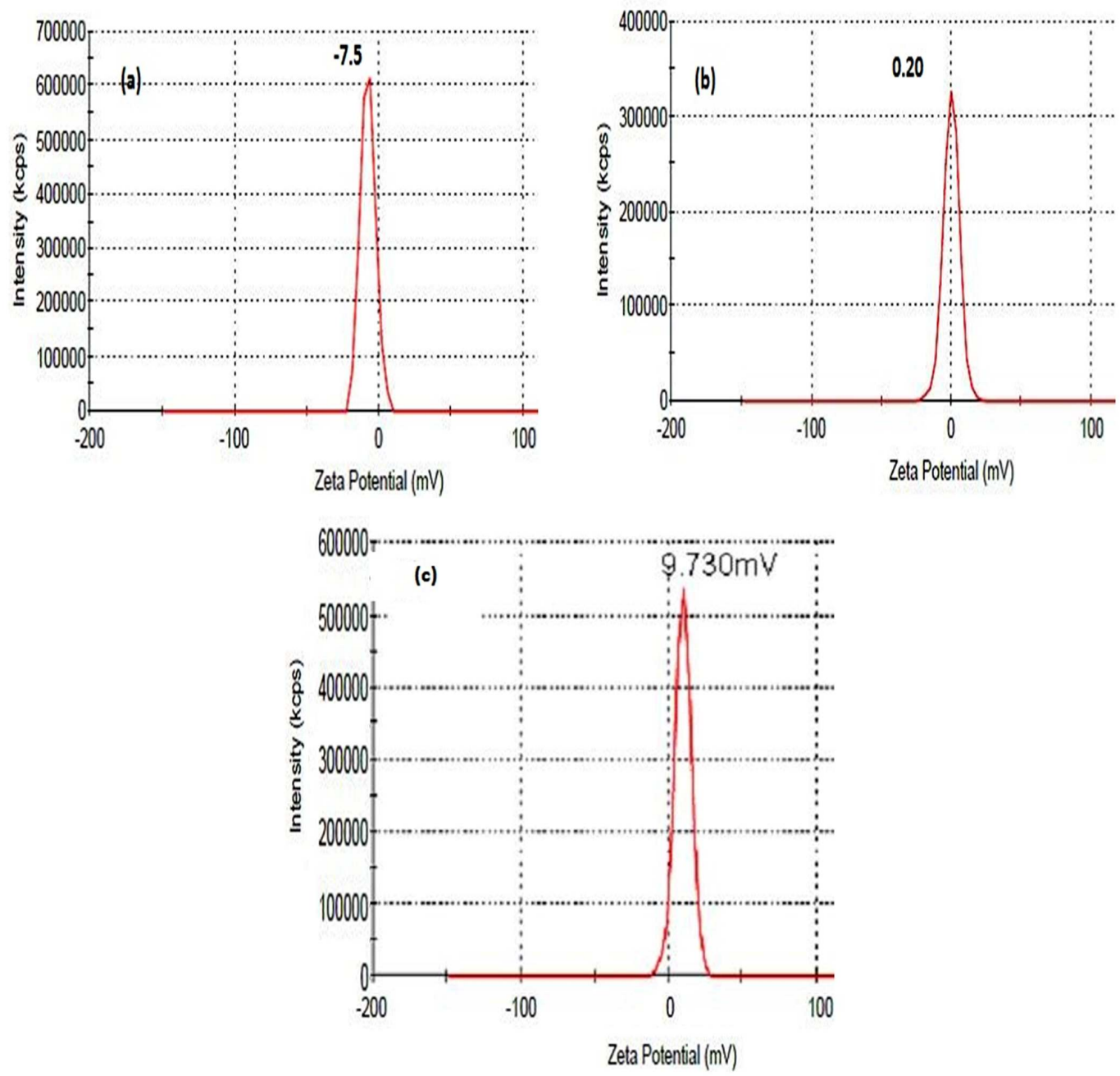

Fig. 4. Zeta potential measuring the dispersion behavior of (a) Ag NPs, (b) $\mathrm{Ag}^{+1}$ based Ag nanocomposite, and (c) $\mathrm{Fe}^{+3}$ based $\mathrm{Ag}$ nanocomposite, respectively.

dispersion of metal nanostructures and delimit the possibilities of coalescence that is a major drawback of metal nanostructures, by which they can lose their desirable properties [17]. In order to improve the dispersion of nano $\mathrm{Ag}$ in any suitable solvent like ethanol, and similarly to decrease its agglomeration propensity, the dispersion trend governed by $\mathrm{PCz}$ was determined by finding zeta potential. The dispersion behavior displayed by zeta potential values of pure $\mathrm{Ag} \mathrm{NPs}, \mathrm{Ag}^{+1}$ and $\mathrm{Fe}^{+3}$ assisted $\mathrm{PCz}$ coated Ag NPs in ethanol is displayed in Fig. 4. The Ag NPs have a propensity to agglomerate due to their high potential energy, which was reduced significantly as denoted by respective data. The dispersion behavior of pure Ag NPs as reflected by zeta potential values is of $-7.5 \mathrm{mV}$. However, it was observed that dispersion of $\mathrm{Ag}$ was increased from -7.5 to 0.2 when it was coated by $\mathrm{PCz}$ by using $\mathrm{Ag}^{+1}$ and to $9.7 \mathrm{mV}$ when it was coated by $\mathrm{Fe}^{+3}$. Therefore, $\mathrm{PCz}$ coating in a composite form was found to be useful to increase its dispersion behavior. The data revealed that the dispersion of nanostructures was enhanced as the polymer thickness was increased. These dispersed composite nanostructures could be immobilized on alumina, nickel or glass substrate to study their analytical applications: in detection of metal ions, $\mathrm{CO}_{2}$ and $\mathrm{N}$ containing bases, such as ammonia, amine, 
and nucleotides. They can also be transformed into a film to investigate the electro/photo conductivity and electric and optical chromic behavior [18].

\section{Conclusion}

The Ag NPs encapsulated by PCz were synthesized in two steps. The first step was a synthesis of Ag NPs by polyol reduction method and the second step was fabrication of $\mathrm{PCz}$ around the Ag NPs by using $\mathrm{Ag}^{+1}$ and $\mathrm{Fe}^{+3}$ as adsorbed oxidants. The morphology of each composite was determined by TEM, which clearly demonstrated the phase of metal NPs and polymer coating. $\mathrm{FeCl}_{3}$ was found to have higher potential to cast the polymer coating as compared to $\mathrm{AgNO}_{3}$. FT-IR confirmed $\mathrm{PCz}$ as polymer by showing $\mathrm{N}-\mathrm{H}, \mathrm{N}-\mathrm{C}$ and ring stretching vibrations. The optical behavior was found to be preserved when NPs were coated by polymer, however, in case of $\mathrm{FeCl}_{3}$, for which the polymer coating was considerably thicker, $\pi-\pi^{*}$ transition was also observed. Similarly, in this study, the dispersion behavior was found significantly increased when NPs were coated by PCz, however, the increase was connected with the increase in polymer thickness. It was more pronounced in case of $\mathrm{FeCl}_{3}$. Therefore, it was concluded that $\mathrm{PCz}$ which possess good optical and dispersion behavior could be tailored around Ag NPs in a composite form and a material ensuring conservation of existing properties along with combining of complementary characteristics. The study could be helpful to explore luminescent, electrochromic behavior, sensing ability and metal atom detection ability of $\mathrm{PCz}$ based $\mathrm{Ag}$ nanocomposite synthesized by $\mathrm{Ag}^{+}$and $\mathrm{Fe}^{+3}$ using as oxidants.

\section{Acknowledgements}

The laboratory facilities and characterization assistance provided by Prof. Dr. Li Xiaoyu and Dr. Qiu Teng to accomplish this work is greatly acknowledged. Moreover, financial assistance provided by the Higher Education Commission (HEC), Pakistan, is particularly acknowledged.

\section{References}

[1] Yasnaya M.A., Kornilov D.Y., Sytnikov E.V., Sinelnikov B.M., Kargin N.I., Khoroshilova S.E., Inorg. Mater, 44 (2008), 230.

[2] Pillai S., Behara R., Nestler H., Suter Marc J.F., Sigg L., SCHIRMER K., PNAS, 111 (2014), 3490.

[3] Aminur M.R., Pankaj K., DeOg-Su P., YoonBo S., Sensors, 8 (2008), 118.

[4] Rose M.M., Karen I.W., Prog. Polym. Sci., 40 (2015), 63.

[5] Matoliukstytea A., Burbulisa E., GrazuleVicius J.V., Gaidelisb V., Jankauskas V., Synth. Met., 158 (2008), 462.

[6] Yadong Z., Tatsuo W., Hiroyuki S., J. Polym. Sci. A-Polym. Chem., 34 (1996), 2289.

[7] Grigoras M., Antonoaia N.C., Eur. Polym. J., 41 (5) ( 2005), 1079.

[8] Ahmad Z., Teng Q., Jiangru Z., Li, X., J. Mater. Sci., 44 (2009), 6054.

[9] Chen A.H., XIE H.X., Wang H.Q., Li H. Y., LI X.Y., Synth. Met., 156 (2006), 346.

[10] Ahmad Z., QIU T., Haiqiao W., Choudhry M.A., Li X., Met. Mater. Int., 17 (3) (2011), 417.

[11] Miyazaki T., Kim S.K., Hoshino K., Chem. Mater., 18 (2006), 5302.

[12] Jain P.K., Huang X., El-SaYed I.E., El-SAYed M.A., Plasmonics, 2 (2007), 107.

[13] Boozer C., Yu Q., Chen S., ChI-Ying L., Homola J., Yee S.S., JiAng S., Sen. Actuators B, 90 (1 - 3) (2003), 22.

[14] Sandee A.J., Williams C.K., Evans N.R., Davies J.E., Boothby C.E., Kohler A., Friend R.H., Holmes A.B., J. Am. Chem. Soc., 126 (22) (2004), 7041.

[15] Zahoor A., Afshan A., Mazher M., Imran A., Rehana A., Muhammad A., J. Nanostruct. Chem., 5 (2015), 325.

[16] Zahoor A., Teng Q., Wang H., Choudhry M.A., LI X., Met. Mater. Int., 17 (3) (2011), 417.

[17] Zhong L.W., Xiangdong F., J. Phys. Chem. B, 107 (2003), 13563.

[18] Bin W., Jinsheng Z., Jun X., Chuansheng C., RENMIN L., Int. J. Electrochem. Sci., 7 (2012) 2781. 